\title{
Genome-wide estimates of inbreeding in unrelated individuals and their association with cognitive ability
}

\author{
Robert A Power ${ }^{\star, 1}$, Craig Nagoshi ${ }^{2}$, John C DeFries ${ }^{3}$, Wellcome Trust Case Control Consortium $2^{4}$ and \\ Robert Plomin ${ }^{1}$
}

The consequence of reduced cognitive ability from inbreeding has long been investigated, mainly restricted to cousin-cousin marriages. Molecular genetic techniques now allow us to test the relationship between increased ancestral inbreeding and cognitive ability in a population of traditionally unrelated individuals. In a representative UK sample of 2329 individuals, we used genome-wide SNP data to estimate the percentage of the genome covered by runs of homozygous SNPs (ROH). This was tested for association with general cognitive ability, as well as measures of verbal and non-verbal ability. Further, association was tested between these traits and specific ROH. Burden of ROH was not associated with cognitive ability after correction for multiple testing, although burden of ROH was nominally associated with increased non-verbal cognitive ability $(P=0.03)$. Moreover, although no individual $\mathrm{ROH}$ was significantly associated with cognitive ability, there was a significant bias towards increased cognitive ability in carriers of ROH $(P=0.002)$. A potential explanation for these results is increased positive assortative mating in spouses with higher cognitive ability, although we found no evidence in support of this hypothesis in a separate sample. Reduced minor allele frequency across the genome was associated with higher cognitive ability, which could contribute to an apparent increase in ROH. This may reflect minor alleles being more likely to be deleterious.

European Journal of Human Genetics (2014) 22, 386-390; doi:10.1038/ejhg.2013.155; published online 17 July 2013

Keywords: intelligence; assortative mating; inbreeding; runs of homozygosity; genome-wide association study; cognitive ability

\section{INTRODUCTION}

Research on consanguineous marriages, and other forms of inbreeding, has long shown a reduction in cognitive abilities in the offspring of such unions. ${ }^{1-3}$ The presumed mechanism is that detrimental recessive mutations are more likely to be identical by descent in the offspring of such unions and so have a greater chance of being expressed. To date, research on the relationship between inbreeding and cognitive ability has largely been restricted to recent inbreeding events as determined by pedigree, although one genome-wide study of ancestral inbreeding has been reported for 10 unrelated families each with two mentally retarded siblings. ${ }^{4}$ It has been suggested that intellectual disability is under negative selection, and that recent deleterious mutations have an important role in the underlying aetiology. ${ }^{5,6}$ The wealth of molecular genetic data currently available allows estimates of inbreeding on a genome-wide level and to examine the effects of long-term ancestral levels of inbreeding. ${ }^{7}$ Such an association with inbreeding, as measured by runs of homozygous polymorphisms ( $\mathrm{ROH})$, has previously been identified with several behavioural traits, such as schizophrenia, Parkinson's disease and personality measures, ${ }^{8-10}$ as well as non-behavioural traits such as height. ${ }^{11}$

The relationship between inbreeding on a population level and cognitive ability is particularly interesting due to assortative mating, non-random mating, which is greater for cognitive ability than for other behavioural traits, as well as physical traits such as height and weight. Positive assortative mating has been reported for cognitive ability, particularly for verbal traits, with spousal correlations generally around $0.5 .^{12-16}$ Assortative mating should lead to greater genetic similarity between mates at causal loci for cognitive ability and to a lesser extent across the genome, which in turn reduces heterozygosity at these loci. In other words, in contrast to the genome-wide reduction of heterozygosity caused by inbreeding, the reduction of heterozygosity due to assortative mating for a trait is limited to loci associated with the trait. However, if many loci affect the trait-which is the conclusion from genome-wide association studies of complex traits and common disorders, including cognitive ability ${ }^{17}, 18$-the trait-specific reduction of heterozygosity might be observed genome-wide even if the effects of individual loci are too small to be observed. Another difference between inbreeding and assortative mating is that the effects of inbreeding are expected to be negative, lowering cognitive ability, whereas the effects of assortative mating affect the high, as well as the low end of the ability distribution, thus increasing genetic variance, that is, when high-ability parents mate assortatively, their children are more likely to be homozygous for variants for high ability, just as offspring of low-ability parents are more likely to be homozygous for variants for low ability.

In this paper, we examine the effects of genome-wide burden of autosomal runs of homozygosity $\left(\mathrm{F}_{\mathrm{ROH}}\right)$ on cognitive ability to test whether the association of reduced ability with extreme inbreeding,

${ }^{1}$ King's College London, MRC Social, Genetic \& Developmental Psychiatry Centre, Institute of Psychiatry, London, UK; ${ }^{2}$ Department of Psychology and School of Social Work, University of Texas-Arlington, Arlington, TX, USA; ${ }^{3}$ Institute for Behavioral Genetics, University of Colorado, Boulder, CO, USA

${ }^{4}$ see Appendices for membership of WTCCC2

*Correspondence: Robert Power, King's College London, MRC Social, Genetic \& Developmental Psychiatry Centre, Institute of Psychiatry, London, SE5 8AF, UK Tel: + 44207848 0873; Fax: + 44207848 0866; E-mail: Robert.r.power@kcl.ac.uk

Received 29 November 2012; revised 10 March 2013; accepted 27 May 2013; published online 17 July 2013 
such as cousin-cousin marriage, is also reflected in a similar association with ancestral levels of relatedness. Although inbreeding is classically defined as an excess of homozygosity, levels of homozygosity are sensitive to population stratification and technical artifacts. By restricting to segments of homozygous markers presumably identical by descent, the use of $\mathrm{F}_{\mathrm{ROH}}$ has previously been shown to best capture ancestral inbreeding. ${ }^{7}$ We also tested for associations between specific $\mathrm{ROH}$ and cognitive ability. Lastly, in a separate sample, we considered the effect of assortative mating on the results of our $\mathrm{F}_{\mathrm{ROH}}$ analysis.

\section{MATERIALS AND METHODS}

\section{Participants}

The Twins Early Development Study (TEDS) recruited over 11000 families of twins born within England and Wales between 1994 and 1996. ${ }^{19,20}$ Comparative analysis of the TEDS sample to census data has shown it to be representative of the UK population in terms of demographics. ${ }^{21}$ Ethical approval was provided by the Institute of Psychiatry, London (05/Q0706/228), and informed consent was obtained from all families. In this analysis, individuals were excluded if they reported severe current medical problems, as well as children who had suffered severe problems at birth or whose mothers had suffered severe problems during pregnancy. Twins whose zygosity was unknown or uncertain or whose first language was not English were also excluded. Finally, analysis was restricted to twins whose parents reported their ethnicity as 'white'. Genotyping on the Affymetrix 6.0 GeneChip and subsequent quality control were carried out as part of the WTCCC2 project for 3154 individuals (one member of a twin pair) for whom cognitive data at age 12 were also available. The methods and quality control have been described in greater detail elsewhere ${ }^{22}$ as part of the WTCCC2 project. $^{23}$ In total, 2329 genotyped individuals were available for whom phenotype information at age 12 were also available.

\section{Cognitive measures}

Verbal and non-verbal tests were administered using web-based testing. ${ }^{24}$ The verbal tests consisted the Similarities subtest and the Vocabulary subtest from the Wechsler Intelligence Scale for Children (WISC-III-UK). ${ }^{25}$ The non-verbal tests were the Picture Completion subtest from the WISC-III-UK and Conceptual Grouping from the McCarthy Scales of Children's Abilities. ${ }^{26}$ A general score was derived from the test battery as the standardized sum of the standardized subtest scores, which correlates 0.99 with a score derived as the first principal component of the test battery score. ${ }^{27}$

\section{Runs of homozygosity}

$\mathrm{F}_{\mathrm{ROH}}$ was defined as the percentage of an individual's genome consisted of runs of homozygosity (ROH). Before analysis, SNPs were pruned for LD within PLINK, removing any SNPs with an $R^{2}$ of 0.90 with any other SNP in a 50 SNP window, leaving 394563 SNPs. $\mathrm{F}_{\mathrm{ROH}}$ was also derived within PLINK, which uses a series of sliding windows across the genome to call $\mathrm{ROH}$ within each individual separately. The size of the windows was set to 65 consecutive SNPs, so any single SNP would be found in 65 different windows. If at least 4 $(5 \%)$ of these windows contained entirely homozygous SNPs, then the SNP in question could be included within a ROH. Within windows, one missing SNP was allowed. To avoid false positives, only $\mathrm{ROH}$ with a minimum of 65 consecutive SNPs covering $2.3 \mathrm{Mb}$ were used when calculating the total proportion of the genome covered by $\mathrm{ROH}$. In addition, the required minimum density in a $\mathrm{ROH}$ was set at $200 \mathrm{~kb}$ per SNP, and the maximum gap between two consecutive homozygous SNPs was set at $500 \mathrm{~kb}$. The $\mathrm{X}$ chromosome was excluded from the analyses. The estimate of the total genome captured was $2.7 \times 10^{9} \mathrm{bp}$. It has been suggested that $\mathrm{F}_{\mathrm{ROH}}$ at these recommended settings estimates $\mathrm{F}$ more accurately than SNP-by-SNP indices and that PLINK captures ROH more accurately than other software. ${ }^{7,28} \mathrm{~F}_{\mathrm{ROH}}$ was tested for association with each of the cognitive variables in a linear regression model. The first 10 ancestry-informative principal components were used as covariates, as was the percentage of SNPs missing for an individual and the traditional measure of inbreeding as an excess of homozygous SNPs compared with the expected number based on Hardy-Weinberg equilibrium. The latter was obtained using the '-het' command in PLINK, ${ }^{29}$ and was used to correct for differences in overall homozygosity resulting from population stratification, DNA quality or other confounders. Sex, age, study cohort and socio-economic status were also used as covariates to remove any subtle differences in $\mathrm{F}_{\mathrm{ROH}}$ they might contribute to. As a further analysis, individual $\mathrm{ROH}$ was tested for association with cognitive traits. This analysis was restricted to those $\mathrm{ROH}$ found for more than 10 individuals and again corrected for the first 10 ancestry-informative principal components. These analyses were performed in STATA.

\section{Assortative mating for cognitive ability}

For reasons described later, we tested whether the strength of assortative mating differs for couples with higher versus lower cognitive ability. Here, we used data from the Hawaii Family Study of Cognition (HFSC), ${ }^{30,31}$ as no such parental data were available for the genotyped individuals in TEDS. The HFSC includes 949 families of Caucasian descent and 400 families of Japanese descent living in Hawaii between the years 1972 and 1976. The cognitive measures in the HFSC included 15 cognitive abilities tests, ${ }^{30-32}$ which yielded 4 factor scores for verbal ability, spatial ability, perceptual speed and accuracy, and visual memory, and a general score based on an unrotated first principal component for the 15 tests. These five measures of cognitive ability were used to stratify both mothers and fathers separately as 0.5 standard deviation below the mean, 0.5 above the mean, and the intermediate group, which roughly divided the sample into thirds. Correlations between parents within the three groups were compared for the five cognitive measures, first using the mothers' measures to stratify couples and then using fathers'.

\section{RESULTS}

Table 1 includes descriptive statistics for $\mathrm{F}_{\mathrm{ROH}}$ and the three measures of cognitive ability (general, verbal, and non-verbal). $\mathrm{F}_{\mathrm{ROH}}$ is slightly positively skewed, as it represents the total percentage of the genome that includes runs of homozygosity $(\mathrm{ROH})$. The Average percentage of genome covered by $\mathrm{ROH}$ was $0.7 \%$ (95\% CI $0.65-0.72 \%)$. Verbal and non-verbal abilities correlate 0.49 ; because general cognitive ability is the sum of the standardized verbal and non-verbal subtests, they correlate much more highly with general ability (0.87 and 0.86 , respectively).

Table 2 presents the results of the linear regression analyses. No significant regression was found between $\mathrm{F}_{\mathrm{ROH}}$ and the cognitive measures after correction for multiple testing, although the association with non-verbal cognitive ability was nominally significant

Table 1 Descriptive statistics for percentage of genome composed of runs of homozygosity $\left(\mathrm{F}_{\mathrm{ROH}}\right)$ and the three cognitive measures for individuals included in analyses $(n=2329)$

Inbreeding indices

Mean number of Mean length of runs

Standard runs of homozyosity of homozygosity in

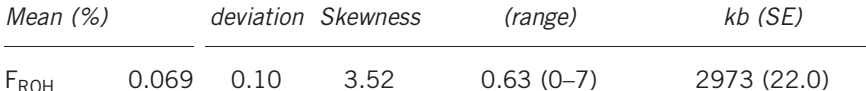

Cognitive abilities

\begin{tabular}{lrcccc} 
Mean & & $\begin{array}{c}\text { Standard } \\
\text { deviation }\end{array}$ & Skewness & $\begin{array}{c}\text { Correlation with } \\
\text { general ability }\end{array}$ & $\begin{array}{c}\text { Correlation with } \\
\text { verbal ability }\end{array}$ \\
\hline General & 0.039 & 0.97 & -0.41 & 1 & - \\
Verbal & 0.017 & 0.98 & -0.62 & 0.87 & 1 \\
Non-verbal & 0.057 & 0.95 & -0.28 & 0.86 & 0.49 \\
\hline
\end{tabular}


$(P=0.03)$. Although this association was not statistically significant, it is noteworthy that every regression in Table 2 is positive, indicating that increased homozygosity tends to be associated with higher cognitive scores across different measures of cognitive ability (general, verbal and non-verbal).

Our analysis identified 87 loci where $\mathrm{ROH}$ overlapped in 10 or more individuals. For these overlapping regions we tested for association with each of the cognitive measures and again showed no significant associations after correction for multiple testing $\left(P\right.$-value of less than $\left.5.7 \times 10^{-4}\right)$. A sign test of the direction of effect across all $\mathrm{ROH}$ showed a disproportionately large number of positive associations, indicating that $\mathrm{ROH}$ are associated with higher cognitive ability $(P=0.002)$. The sign test was non-significant for verbal ability but highly significant for non-verbal ability $\left(P<10^{-6}\right)$. The sign test for non-verbal ability alone remained significant after correcting for an individual's genome-wide $\mathrm{F}_{\mathrm{ROH}}$ score $\left(P<10^{-6}\right)$.

As explained earlier, positive assortative mating can also lead to genome-wide homozygosity for trait-specific loci, and, unlike inbreeding, assortative mating can affect the high as well as the low end of the ability distribution. One possible explanation for the trend suggesting a positive correlation between homozygosity and cognitive scores in our data is that positive assortative mating on intelligence might be greater for higher cognitive ability individuals. This greater phenotypic similarity within higher cognitive ability parents would presumably result in increased genetic similarity at loci associated with cognitive ability, and would lead to a positive correlation between homozygosity and cognitive performance in their offspring. As the genotyped TEDS sample lacked parental data, we used a separate sample, the Hawaii Family Study of Cognition (HFSC), to analyse spousal correlations for high, intermediate and low cognitive ability individuals. Table 3 summarizes the results of analyses

Table 2 Results of the association between percentage of genome composed of runs of homozygosity $\left(\mathrm{F}_{\mathrm{ROH}}\right)$ and the three cognitive measures (general, verbal and non-verbal)

\begin{tabular}{lccc}
\hline & Coefficient & Standard error & P-value \\
\hline General cognitive ability & 34.1 & 27.8 & 0.17 \\
Verbal cognitive ability & 2.4 & 25.6 & 0.92 \\
Non-verbal cognitive ability & 56.7 & 25.3 & 0.03 \\
\hline
\end{tabular}

investigating whether assortative mating differs as a function of cognitive ability and shows that assortative mating for general cognitive ability was similar for low and high ability individuals of both ethnic groups (Japanese Americans and European Americans). Similar results were found for the four measures of specific cognitive abilities (Supplementary Table 1).

As a final analysis, we returned to the genome-wide genotype data used in the $\mathrm{F}_{\mathrm{ROH}}$ analysis to test whether the positive association between $\mathrm{F}_{\mathrm{ROH}}$ and cognitive ability might reflect reduced SNP minor allele frequencies in high cognitive ability individuals. As the minor allele frequency gets closer to zero, it increases the probability of the SNP being homozygous by chance for the major allele. If lower minor allele frequencies were a marker for increased cognitive abilityperhaps because minor alleles are more likely to be deleterious or population stratification-then it could lead to a spurious positive association between homozygosity and cognitive ability. Using the minor allele frequency of each SNP, we performed a sign test comparing the frequency in those individuals in the top quartile for non-verbal cognitive ability to the frequency of the same SNP in a sample of those in the bottom quartile (see Supplementary Table 2). The high cognitive ability sample was found to be significantly associated with lower minor allele frequencies across all 394563 SNPs after pruning for linkage disequilibrium $(P=0.04$ for a sign test between cohorts of high and low ability individuals), though the difference in average minor allele frequency across all SNPs was tiny ( 0.21823 to 0.21824 , unpaired $t$-test non-significant).

\section{DISCUSSION}

Our results show that within a representative UK population sample there was a weak nominally significant association between burden of autosomal runs of homozygosity and higher non-verbal cognitive ability. This nominal association with increased cognitive ability is counterintuitive when compared with the results from more extreme inbreeding based on pedigree information. ${ }^{1-3}$ A potential explanation for this direction of effect is that individuals with higher cognitive ability might show greater positive assortative mating, which would lead to increased homozygosity at loci for higher cognitive ability in their offspring. However, in a separate sample we showed that greater positive assortative mating was not associated with higher cognitive ability. While these findings seem to provide clear evidence against this hypothesis, it is possible that the genome-wide genetic findings reflect historical mating habits that no longer exist today. It should

Table 3 Assortative mating correlations between parents on general cognitive ability for two ethnic groups in the Hawaiian Family Study of Cognition, European Americans and Japanese Americans

\begin{tabular}{|c|c|c|c|c|c|c|}
\hline \multirow{2}{*}{$\begin{array}{l}\text { Parental correlation stratified by: } \\
\text { European American individuals ( } 949 \text { families) }\end{array}$} & \multicolumn{3}{|c|}{ Father's cognitive ability } & \multicolumn{3}{|c|}{ Mother's cognitive ability } \\
\hline & Low & Middle & High & Low & Middle & High \\
\hline First principal component of measures & 0.13 & 0.11 & 0.05 & 0.13 & 0.12 & 0.11 \\
\hline $95 \%$ confidence intervals & $0.011-0.244$ & -0.001 to 0.219 & -0.065 to 0.165 & $0.004-0.254$ & $0.014-0.224$ & $-0.003-0.225$ \\
\hline Standard deviation & 0.988 & 0.904 & 0.891 & 0.839 & 0.869 & 0.786 \\
\hline Parental correlation stratified by: & \multicolumn{3}{|c|}{ Father's cognitive ability } & \multicolumn{3}{|c|}{ Mother's cognitive ability } \\
\hline Japanese American individuals (400 families) & low & medium & high & low & medium & high \\
\hline First principal component of measures & 0.08 & 0.07 & 0.06 & 0.12 & 0.10 & 0.11 \\
\hline $95 \%$ confidence intervals & $-0.130-0.289$ & $-0.117-0.245$ & $-0.133-0.247$ & $-0.094-0.324$ & $-0.090-0.273$ & $-0.080-0.291$ \\
\hline Standard deviation & 0.878 & 0.938 & 0.899 & 1.040 & 0.989 & 0.952 \\
\hline
\end{tabular}

Each sample was divided roughly into thirds based on cognitive ability: low ( $>0.5$ standard deviation below the mean), middle ( 0.5 SD below to 0.5 SD above mean), and high ( $>0.5$ standard deviation above the mean). The parental correlations were then calculated within each band of cognitive ability. This was done stratifying on both paternal and maternal cognitive ability separately, which may have differed. 
also be noted that there was a reduction in the standard deviations for spousal correlations in the increased cognitive ability groups by an average of $6 \%$ compared with the decreased cognitive ability group (see Table 3), which could reflect lesser genetic variability in the high ability couples or a ceiling effect on the cognitive tests. This lesser phenotypic variability at the high ability end would have a small effect in reducing the spouse correlations and potentially confound our analysis.

Genetic variants were found to have a slight (though significant) reduction in minor allele frequency across the genome in individuals in the top quartile of general cognitive ability compared with those in the bottom quartile (means of 21823 and 21824), which in turn could lead to increased homozygosity by chance. This could indicate that these individuals descend from subtly different ancestral populations that experienced loss of variation. This difference in ancestry may be correlated with either genetic variants for improved cognitive ability, or with social or environmental backgrounds that lead to higher cognitive ability, though our analysis of $\mathrm{F}_{\mathrm{ROH}}$ corrected for socio-economic status and population stratification. Another potential explanation is that the reduced minor allele frequency in the high cognitive ability is reflective of the less frequent allele being deleterious due to selection against it. As a result, these high-functioning individuals could benefit from having more major alleles at fixation and a reduced burden of rarer deleterious variants.

Overall, these results highlight the importance of understanding mating habits, such as inbreeding and assortative mating, when investigating the genetic architecture of complex traits such as cognitive ability. The results certainly suggest that there is no large effect of $\mathrm{F}_{\mathrm{ROH}}$ on reduced cognitive ability, the expected direction of effect. The nominally significant associations found in this study may even suggest that in the case of non-verbal cognitive ability, beneficial associations with homozygosity at specific loci might outweigh the negative effects of genome-wide inbreeding and that the relationship between inbreeding and cognitive ability may be more complicated than previously thought.

\section{CONFLICT OF INTEREST}

The authors declare no conflict of interest.

\section{ACKNOWLEDGEMENTS}

Twins Early Development Study (TEDS) is supported by a program grant to RP from the UK Medical Research Council [G0901245; and previously G0500079], with additional support from the US National Institutes of Health [HD044454; HD046167; HD059215]. Genome-wide genotyping was made possible by grants from the Wellcome Trust Case Control Consortium 2 project [085475/B/08/Z; 085475/Z/08/Z]. RP is supported by a Medical Research Council Research Professorship award [G19/2] and a European Research Council Advanced Investigator award [295366]

1 Morton NE: Effect of inbreeding on IQ and mental retardation. Proc Nat/ Acad Sci USA 1978; 75: 3906-3908.

2 Woodley MA: Inbreeding depression and IQ in a study of 72 countries. Intelligence 2009; 37: 268-276.

3 Jensen AR: Effects of inbreeding on mental-ability factors. Personality and Individual Differences 1983; 4: 71-87.
4 Schuurs-Hoeijmakers JH, Hehir-Kwa JY, Pfundt R et al: Homozygosity mapping in outbred families with mental retardation. Eur J Hum Genet 2011; 19: 597-601.

5 Guilmatre A, Dubourg C, Mosca AL et al: Recurrent rearrangements in synaptic and neurodevelopmental genes and shared biologic pathways in schizophrenia, autism, and mental retardation. Arch Gen Psychiatry 2009; 66: 947-956.

6 Vissers LE, de Ligt J, Gilissen C et al: A de novo paradigm for mental retardation. Nat Genet 2010; 42: 1109-1112.

7 Keller MC, Visscher PM, Goddard ME: Quantification of inbreeding due to distant ancestors and its detection using dense single nucleotide polymorphism data. Genetics 2011; 189: 237-249.

8 Verweij KJ, Yang J, Lahti J et al: Maintenance of genetic variation in human personality: testing evolutionary models by estimating heritability due to common causal variants and investigating the effect of distant inbreeding. Evolution 2012; 66 : 3238-3251.

9 Keller MC, Simonson MA, Ripke S et al: Runs of homozygosity implicate autozygosity as a schizophrenia risk factor. PLoS Genet 2012; 8: e1002656.

10 Simon-Sanchez J, Kilarski LL, Nalls MA et al: Cooperative genome-wide analysis shows increased homozygosity in early onset Parkinson's disease. Plos One 2012; 7: e28787.

11 McQuillan R, Eklund N, Pirastu N et al: Evidence of inbreeding depression on human height. PLoS Genet 2012; 8: e1002655.

12 Vandenburg SG: Assortative mating, or who marries whom? Behav Genet 1972; 2: 127-157.

13 Reynolds CA, Baker LA, Pedersen NL: Multivariate models of mixed assortment: phenotypic assortment and social homogamy for education and fluid ability. Behav Genet 2000; 30: 455-476

14 van Leeuwen M, van den Berg SM, Boomsma DI: A twin-family study of general IQ. Learning and Individual Differences 2008; 18: 76-88.

15 Mascie-Taylor CG: Spouse similarity for IQ and personality and convergence. Behav Genet 1989; 19: 223-227.

16 Loehlin JC: Heredity-environment analyses of Jencks's IQ correlations. Behav Genet 1978; 8: 415-436.

17 Davies G, Tenesa A, Payton A et al: Genome-wide association studies establish that human intelligence is highly heritable and polygenic. Mol Psychiatry 2011; 16: 996-1005.

18 Davis OS, Butcher LM, Docherty SJ et al: A three-stage genome-wide association study of general cognitive ability: hunting the small effects. Behav Genet 2010; 40: 759-767.

19 Trouton A, Spinath FM, Plomin R: Twins early development study (TEDS): a multivariate, longitudinal genetic investigation of language, cognition and behavior problems in childhood. Twin Res 2002; 5: 444-448.

20 Oliver BR, Plomin R: Twins' Early Development Study (TEDS): a multivariate, longitudinal genetic investigation of language, cognition and behavior problems from childhood through adolescence. Twin Res Hum Genet 2007; 10: 96-105.

21 Kovas Y, Haworth CM, Dale PS, Plomin R: The genetic and environmental origins of learning abilities and disabilities in the early school years. Monogr Soc Res Child Dev 2007; 72: vii 1-144.

22 Plomin R, Davis OSP: Common DNA markers can account for at least half of the genetic influence on cognitive abilities. Psychol Sci 2013; 24: 562-568.

23 Barrett JC, Lee JC, Lees CW et al: Genome-wide association study of ulcerative colitis identifies three new susceptibility loci, including the HNF4A region. Nat Genet 2009; 41: 1330-1334.

24 Davis OS, Haworth CM, Plomin R: Learning abilities and disabilities: generalist genes in early adolescence. Cognitive neuropsychiatry 2009; 14: 312-331.

25 Wechsler D: Wechsler intelligence scale for children-, third edition UK (WISC-IIIUK) Manual, London, UK: The Psychological Corporation 1992

26 McCarthy D: McCarthy scales of children's abilities. New York, NY, USA: The Psychological Corporation, 1972.

27 Davis OSP, Arden R, Plomin R: g in middle childhood: Moderate genetic and shared environmental influence using diverse measures of general cognitive ability at 7,9 and 10 years in a large population sample of twins. Intelligence 2008; 36: 68-80.

28 Howrigan DP, Simonson MA, Keller MC: Detecting autozygosity through runs of homozygosity: a comparison of three autozygosity detection algorithms. Bmc Genomics 2011; 12: 460.

29 Purcell S, Neale B, Todd-Brown K et al: PLINK: a tool set for whole-genome association and population-based linkage analyses. Am J Hum Genet 2007; 81: 559-575.

30 DeFries JC, Johnson RC, Kuse AR et al: Familial resemblance for specific cognitive abilities. Behav Genet 1979; 9: 23-43.

31 Wilson JR, De Fries JC, McClearn GE, Vanderberg SG, Johnson RC, Rashad MN: Cognitive abilities: use of family data as a control to assess sex and age differences in two ethnic groups. Int J Aging Hum Dev 1975; 6: 261-276.

32 Nagoshi CT, Johnson RC, DeFries JC, Wilson JR, Vandenberg SG: Group differences and first principal-component loadings in the Hawaii family study of cognition: a test of the generality of 'Spearman's hypothesis'. Personality and Individual Differences 1984; 5: 751-753.

Supplementary Information accompanies this paper on European Journal of Human Genetics website (http://www.nature.com/ejhg) 


\section{APPENDICES}

Membership of Wellcome Trust Case Control Consortium 2

\section{Management Committee}

Peter Donnelly (Chair) ${ }^{1,2}$, Ines Barroso (Deputy Chair) ${ }^{3}$, Jenefer M Blackwell ${ }^{4,5}$, Elvira Bramon ${ }^{6}$, Matthew A Brown ${ }^{7}$, Juan P Casas ${ }^{8}$, Aiden Corvin ${ }^{9}$, Panos Deloukas ${ }^{3}$, Audrey Duncanson ${ }^{10}$, Janusz Jankowski ${ }^{11}$, Hugh S Markus ${ }^{12}$, Christopher G Mathew ${ }^{13}$, Colin NA Palmer ${ }^{14}$, Robert Plomin ${ }^{15}$, Anna Rautanen ${ }^{1}$, Stephen J Sawcer ${ }^{16}$, Richard C Trembath ${ }^{13}$, Ananth C Viswanathan ${ }^{17}$, Nicholas W Wood ${ }^{18}$

\section{Data and Analysis Group}

Chris C A Spencer ${ }^{1}$, Gavin Band ${ }^{1}$, Céline Bellenguez ${ }^{1}$, Colin Freeman ${ }^{1}$, Garrett Hellenthal ${ }^{1}$, Eleni Giannoulatou ${ }^{1}$, Matti Pirinen ${ }^{1}$, Richard Pearson ${ }^{1}$, Amy Strange ${ }^{1}$, Zhan Su ${ }^{1}$, Damjan Vukcevic ${ }^{1}$, Peter Donnelly ${ }^{1,2}$

\section{DNA, Genotyping, Data QC and Informatics Group}

Cordelia Langford ${ }^{3}$, Sarah E Hunt ${ }^{3}$, Sarah Edkins ${ }^{3}$, Rhian Gwilliam ${ }^{3}$, Hannah Blackburn ${ }^{3}$, Suzannah J Bumpstead ${ }^{3}$, Serge Dronov ${ }^{3}$, Matthew Gillman ${ }^{3}$, Emma Gray ${ }^{3}$, Naomi Hammond ${ }^{3}$, Alagurevathi Jayakumar ${ }^{3}$, Owen $\mathrm{T} \mathrm{McCann}^{3}$, Jennifer Liddle ${ }^{3}$, Simon C Potter ${ }^{3}$, Radhi Ravindrarajah ${ }^{3}$, Michelle Ricketts ${ }^{3}$, Matthew Waller ${ }^{3}$, Paul Weston ${ }^{3}$, Sara Widaa ${ }^{3}$, Pamela Whittaker ${ }^{3}$, Ines Barroso ${ }^{3}$, Panos Deloukas ${ }^{3}$.

\section{Publications Committee}

Christopher G Mathew (Chair) $^{13}$, Jenefer M Blackwell ${ }^{4,5}$, Matthew A Brown ${ }^{7}$, Aiden Corvin ${ }^{9}$, Chris C A Spencer ${ }^{1}$

${ }^{1}$ Wellcome Trust Centre for Human Genetics, University of Oxford, Roosevelt Drive, Oxford OX3 7BN, UK; ${ }^{2}$ Department of Statistics, University of Oxford, Oxford OX1 3TG, UK; ${ }^{3}$ Wellcome Trust Sanger Institute, Wellcome Trust Genome Campus, Hinxton, Cambridge
CB10 1SA, UK; ${ }^{4}$ Telethon Institute for Child Health Research, Centre for Child Health Research, University of Western Australia, 100 Roberts Road, Subiaco, Western Australia 6008; ${ }^{5}$ Cambridge Institute for Medical Research, University of Cambridge School of Clinical Medicine, Cambridge CB2 0XY, UK; ${ }^{6}$ Department of Psychosis Studies, NIHR Biomedical Research Centre for Mental Health at the Institute of Psychiatry, King's College London and The South London and Maudsley NHS Foundation Trust, Denmark Hill, London SE5 8AF, UK; ${ }^{7}$ University of Queensland Diamantina Institute, Brisbane, Queensland, Australia; ${ }^{8}$ Department Epidemiology and Population Health, London School of Hygiene and Tropical Medicine, London WC1E 7HT and Department Epidemiology and Public Health, University College London WC1E 6BT, UK; ${ }^{9}$ Neuropsychiatric Genetics Research Group, Institute of Molecular Medicine, Trinity College Dublin, Dublin 2, Eire; ${ }^{10}$ Molecular and Physiological Sciences, The Wellcome Trust, London NW1 2BE, UK; ${ }^{11}$ Department of Oncology, Old Road Campus, University of Oxford, Oxford OX3 7DQ, UK, Digestive Diseases Centre, Leicester Royal Infirmary, Leicester LE7 7HH, UK and Centre for Digestive Diseases, Queen Mary University of London, London E1 2AD, UK; ${ }^{12}$ Clinical Neurosciences, St George's University of London, London SW17 0RE; ${ }^{13}$ King's College London Dept Medical and Molecular Genetics, King's Health Partners, Guy's Hospital, London SE1 9RT, UK; ${ }^{14}$ Biomedical Research Centre, Ninewells Hospital and Medical School, Dundee DD1 9SY, UK; ${ }^{15}$ King's College London Social, MRC Genetic and Developmental Psychiatry Centre, Institute of Psychiatry, Denmark Hill, London SE5 8AF, UK; ${ }^{16}$ University of Cambridge Dept Clinical Neurosciences, Addenbrooke's Hospital, Cambridge CB2 0QQ, UK; ${ }^{17}$ NIHR Biomedical Research Centre for Ophthalmology, Moorfields Eye Hospital NHS Foundation Trust and UCL Institute of Ophthalmology, London EC1V 2PD, UK; ${ }^{18}$ Dept Molecular Neuroscience, Institute of Neurology, Queen Square, London WC1N 3BG, UK. 